Document downloaded from:

http://hdl.handle.net/10251/65709

This paper must be cited as:

Müller, A.; Sanabria-Codesal, E.; Moldoveanu, A.; Asavei, V.; Favennec, J. (2015). On the sum of the transmission and reflection coefficient on the Smith chart and 3D Smith chart. Asia-Pacific Microwave Conference (APMC 2015). IEEE. doi:10.1109/APMC.2015.7411635.

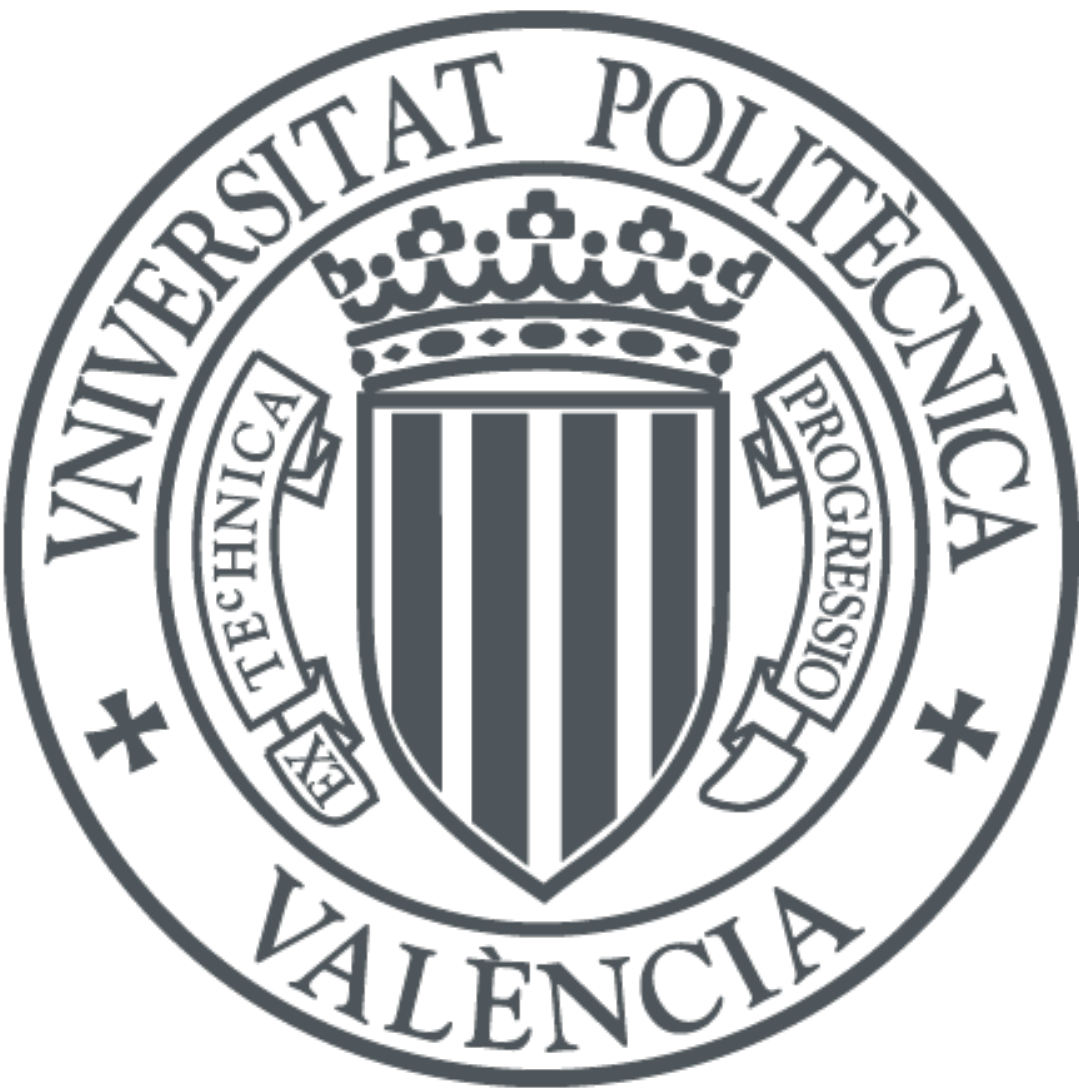

The final publication is available at

http://dx.doi.org/10.1109/APMC.2015.7411635

Copyright IEEE

Additional Information

(C) 2015 IEEE. Personal use of this material is permitted. Permission from IEEE must be obtained for all other uses, in any current or future media, including reprinting/republishing this material for advertising or promotional purposes, creating new collective works, for resale or redistribution to servers or lists, or reuse of any copyrighted component of this work in other works. 


\title{
On the sum of the reflection and transmission coefficient on the Smith chart and 3D Smith chart
}

\author{
A. A. Muller ${ }^{1}$, E. Sanabria-Codesal ${ }^{2}$, A. Moldoveanu ${ }^{3}$, V. Asavei ${ }^{3}$, J.F. Favennec ${ }^{4}$ \\ ${ }^{1}$ Microwave Applications Group, Universitat Politècnica de València - Valencia, 46022 Spain \\ ${ }^{2}$ Departamento de Matemática Aplicada, Universitat Politècnica de València, Valencia, 46022 Spain \\ ${ }^{3}$ Autmomatic Faculty, Politehnica University Bucharest, Romania \\ ${ }^{4}$ Université de Brest, Lab-STICC UMR CNRS 6285, 6 Av Le Gorgeu, CS 93837, 29238 Brest Cedex 3 France
}

\begin{abstract}
The paper presents in premiere a simple mapping property of the sum of the reflection and transmission parameters of reciprocal two port networks. It is proved that although the reflection and transmission parameter may have very complicated paths on the Smith chart, their sum will be always moving on the unit circle if the circuit is symmetric and lossless. Further once symmetrical losses at the ports occur their sum path will switch on a family of circles through one point. Using inversive geometry we construct a new function which maps this family of circles in lines on the extended Smith chart. The proposed method for checking the symmetry uses just two parameters and avoids testing the phase of the corresponding input and output parameters. By means of the 3D Smith chart we propose in the end an alternative approach to visualize the parameters.
\end{abstract}

\section{INTRODUCTION}

In the recent years it was shown that elements of group theory present in geometry may bring to light new tools in microwave engineering [1-4] like the 3D Smith chart, while in [5-6] it was also lately demonstrated how new circle boundaries obtained by ingenious manipulation of different functions of the scattering parameters may still help in the microwave design. Nevertheless in [3] it was proved that the well known unilateral transducer power gain circles are a family of Apollonius circles recently introduced too in microwave engineering in [7]. In this work we prove that the sum of the reflection and transmission coefficient of a traditional symmetrical reciprocal two port network with symmetrical losses at the input and output ports when visualized on the Smith chart, moves on a circular path. The presence of symmetrical losses at the ports influences its position in the family of circles through one point (unit circle when there is no loss). This family of circles may be converted by the usage of inversive geometry [8] into lines, thus it will be shown that the presence of losses in a circuit or asymmetry may be detected by examining the sum of the reflection and transmission coefficient at a single port.

\footnotetext{
II. THE PATH OF THE SUM OF THE TRANSMISSION AND REFLECTION SCATTERING PARAMETERS ON THE SMITH CHART
}

Based on [9], the relationship between the reflection and transmission coefficient $S_{I I}(\omega)$ and $S_{2 I}(\omega)$ (where $\omega$ represents the angular frequency) of a symmetrical two port network may be simplified when expressed in terms of the ABCD chain parameters as (1)-(2) since $(A=D)$. The sum of them $(S(\omega)=$ $\left.S_{I I}(\omega)+S_{2 I}(\omega)\right)$ may be expressed as (3) considering the network reciprocal $(A D-B C=1)$ where $Z_{0}$ is the characteristic impedance of the input and output port.

$$
S_{11}(\omega)=\frac{B(\omega)-C(\omega) Z_{0}{ }^{2}}{2 * A(\omega) Z_{0}+B(\omega)+C(\omega) Z_{0}{ }^{2}}
$$

$$
S_{21}(\omega)=\frac{2 Z_{0}}{2 * A(\omega) Z_{0}+B(\omega)+C(\omega) Z_{0}^{2}}
$$

$$
S(\omega)=S_{21}(\omega)+S_{11}(\omega)=\frac{B(\omega)+Z_{0}(1-A(\omega))}{B(\omega)+Z_{0}(-1+A(\omega))}
$$

In the case of a traditional lossless network $A(\omega)$ will be always real while $B(\omega)$ will be always purely imaginary [10] thus of form $j B_{I}(\omega)$. In this situation it can be seen that the path of $S(\omega)$ in the reflection coefficient's plane will be on a the unit circle since (4) takes place.

$$
|S(\omega)|=\left|\frac{B(\omega)+Z_{0}(1-A(\omega))}{B(\omega)+Z_{0}(-1+A(\omega))}\right|=\left|\frac{j B_{1}(\omega)+Z_{0}(1-A(\omega))}{j B_{1}(\omega)+Z_{0}(-1+A(\omega))}\right|=1
$$

It may be further proved that (3) always represents a family of curves of "coaxal" circles [8] passing through $(1,0)$ when symmetrical losses occur at the ports. Actually it may be demonstrated that (3) can be written as (5), where $F(\omega)$ (5) will be of form $a+j * b(\omega)$ or $a(\omega)+j^{*} b$ thus $S(\omega)$ will have always the form of a Möbius transformation of the extended line $a+j b(\omega)$ or $a(\omega)+j * b$ ( when symmetrical losses at the ports occur) and will map $F(\omega)$ in a family of circles $[1,8]$.

$$
S(\omega)=1-\frac{2}{1+F(\omega)}, \quad F(\omega)=\frac{B(\omega)}{(A(\omega)-1) Z_{0}}
$$

\section{CIRCUITS EXAMPLES}

Let us consider the case of a third order ideal bandpass filter as in Fig. 1 with inductors $L_{1}=L_{3}=6.506 \mathrm{nH}$, capacitors 
$C_{I}=C_{3}=0.9888 \mathrm{pF}$ and with inductor $L_{2}=1.391 \mathrm{nH}$ while capacitor $C_{2}=4.625 \mathrm{pF}$. The input and output characteristic impedances are considered $Z_{0}=50 \Omega$ while the resistances $R_{I}=R_{2}=0$ in an ideal case. This will generate a Chebyshev filter response with a central frequency of $1.984 \mathrm{GHz}$, a bandwidth of $0.5 \mathrm{GHz}$ and a ripple of $0.001 \mathrm{~dB}$.

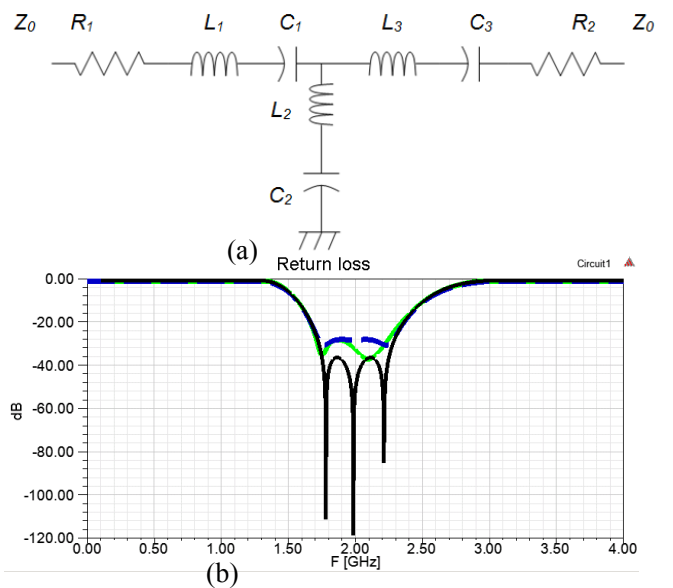

Figure 1.(a) A third order bandpass filter topology.(b) Return loss of the filter for $L_{1}=6.506 \mathrm{nH}, C_{I}=C_{3}=0.9888 \mathrm{pF} L_{2}=1.391 \mathrm{nH}, C_{2}=4.625 \mathrm{pF}, R_{I}=0$ if $\left(L_{3}=\right.$ $6.506 \mathrm{nH}$ and $R_{2}=0$ - dark- ideal response), $\left(L_{3}=6.3 \mathrm{nH}\right.$ and $R_{2}=0$ - green response), $\left(L_{3}=6.506 \mathrm{nH}\right.$ and $R_{2}=2 \Omega$ - blue). Since $\left|\mathrm{S}_{11}\right|=\left|\mathrm{S}_{22}\right|$ in all the cases it is impossible to detect the cause of the perfect matching failure without visualizing the phase of the $S_{11}$ and $S_{22}$ parameters.

In the design of the filter we may fail to achieve the desired values, as for example in the Fig.1.

Let us consider that we design the filter with the preferred values but that we don't succeed to have $R_{I}=R_{2}=0$. Supposing $R_{1}=R_{2}=R$ where $0 \leq R \leq 10 \Omega$ (with a step of $1 \Omega$ ). $S(\omega)$ for the circuit is of form (5) where $F(\omega)$ has the form (6) for the circuit in Fig.1. It is obvious that (6) is an extended line passing through $R_{l}$ (since it's real part does not depend of $\omega$ ) thus $S(\omega)$ which has form (5) will represent a family of circles through $(1,0)$.

$$
F(\omega)=R_{1}+j \frac{\frac{-1}{C_{1}}-\frac{2}{C_{2}}+\left(L_{1}+2 L_{2}\right) \omega^{2}}{\omega}
$$

We may alternatively represent the sum of the transmission and reflection parameter (5) or we may consider the representation of (7) which will map (5) into a family of lines passing through infinity but still perpendicular on the real axes of the reflection coefficient's plane [8]- since each family of coaxal circles through one point $(1,0)$ may mapped into a family of lines :

$$
S_{L}(\omega)=\frac{1}{S(\omega)-1}
$$

By inspection of Fig.2 or Fig. 3 it can be seen that we have to deal with symmetrical circuits with additional symmetrical losses at the input and output ports since (5) is represented by a coaxial family of circles or (7) by a family of lines.

Considering now the circuit in Fig.1 with $L_{l}=6.506 \mathrm{nH}$, $C_{I}=C_{3}=0.9888 \mathrm{pF}, L_{2}=1.391 \mathrm{nH}, C_{2}=4.625 \mathrm{pF}$ while $\mathrm{L}_{3}=6.3 \mathrm{nH}$ and $R_{1}=R_{2}=0$, the sum of the reflection and insertion parameter will not have the form (5) anymore (thus not a circle shape) since $L_{3}$ is now slightly different from $L_{l}$ ( Fig.4). The failure to obtain a symmetrical circuit can be directly detected by examining Fig.4, because the sum of the reflection and transmission parameter doesn't have a circular trajectory anymore as the frequency changes.

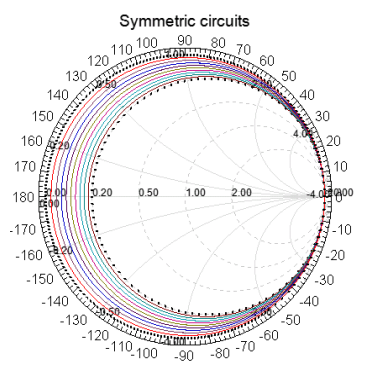

Figure 2. Representation of the sum of the reflection and transmission moves on a family of circles for a symmetrical reciprocal two port network passing through the point $(1,0)$, the representation is done for the Fig.1 considering $L_{l}=L_{3}=6.506 \mathrm{nH}, C_{I}=C_{3}=0.9888 \mathrm{pF}$ and with $L_{2}=1.391 \mathrm{nH}$ while $C_{2}=4.625 \mathrm{pF}$ and $R_{l}=R_{2}=R$. It is considered that $0 \leq R \leq 10$.

(a)

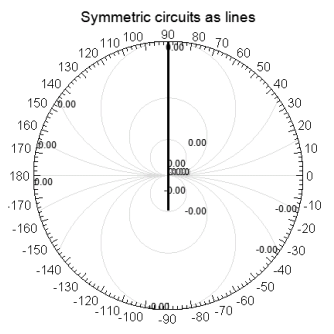

Figure 3. Representation of (7) for a symmetrical reciprocal two port network will be always a generalized lines family passing through infinity. The representation is done for Fig. 1 considering $L_{I}=L_{3}=6.506 \mathrm{nH}, C_{I}=C_{3}=0.9888 \mathrm{pF}$ and with $L_{2}=1.391 \mathrm{nH}$ while $C_{2}=4.625 \mathrm{pF}$ and $R_{l}=R_{2}=R$. It is considered that $0 \leq R \leq 10$ (a) Representation on an extended 2D Smith chart without zooming may seem to map all of them in the same line, (b)zooming the representation we may see the family of lines.

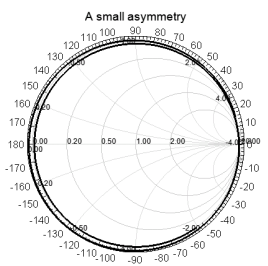

Figure 4. Representation of the sum of the reflection and transmission coefficient for the circuit in Fig. $1 S(\omega)$ when $L_{I}=6.506 \mathrm{nH}, C_{I}=C_{3}=0.9888 \mathrm{pF}$ $L_{2}=1.391 \mathrm{nH}, L_{3}=6.3 \mathrm{nH}, C_{2}=4.625 \mathrm{pF}$ and $R_{I}=R_{2}=0$, the small asymmetry generated by $L_{1} \neq L_{3}$ generates a non circular shape, since it will not have the form (5) anymore.

\section{CIRCUITS EXAMPLES ON THE 3D SMITH CHART}

The sum of the insertion and return loss parameters or (7) may sometimes difficult to be visualized in 2D. $S$ may be in cases of symmetric reciprocal active devices with negative 
resistances mapped into a circle outside of the Smith chart, while $S_{L}$ (7) which represents an extended line to infinity is very difficult to be visualized in $2 \mathrm{D}$, different zooming problems occurring as in Fig. 3.

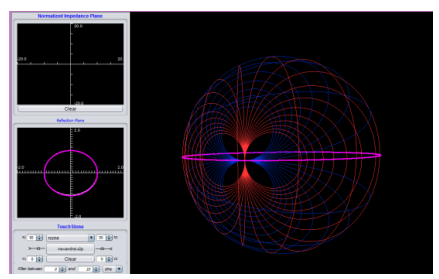

Figure 5. Representation of (5) for the filter topology in Fig.1 and for the same parameters as in Fig. 2 with $R_{I}=R_{2}=0.0-10 \mathrm{GHz}$-symmetrical circuit.

On the 3D Smith chart these visualization problems never occur [1-4]. Mapping the new defined parameters (3) and (7) on the 3D Smith chart with the same stereographical projection used in [1], ensures that these will be always circles (since extended lines are mapped into circles on the Riemann sphere).We may actually see that plotting (3) and (7) on the 3D Smith chart for the symmetrical circuit presented in Fig. 1 and considering (for example $R_{l}=R_{2}=0$ ) we can get Fig.5 and Fig.6, thus circles on the $3 \mathrm{D}$ Smith chart (thus the circuits are symmetric.

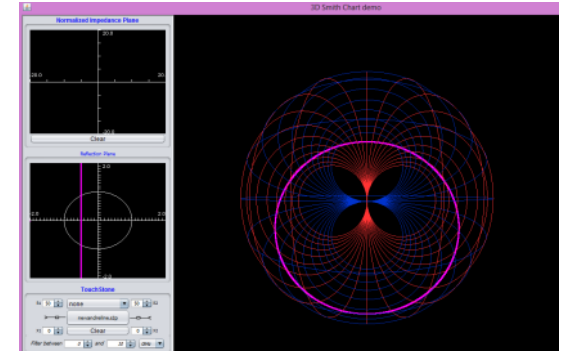

Figure 6. Representation of $S_{L}(7)$ for the filter topology in Fig.1 and the same parameters as in Fig.2- Fig. 3 but with $R_{I}=R_{2}=0$. (the simulation is done in the $0-10 \mathrm{GHz}$ range).

In Fig. 7 we can see the representation of $S(\omega)$ for the circuit in Fig. 1 for the same values as in Fig.4- the presence of the asymmetry may be directly detected on the 3D Smith chart where different zooming scales may be used.

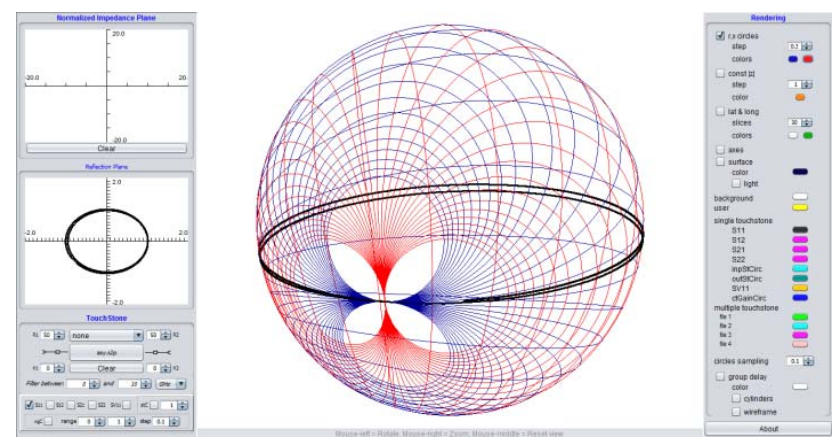

Figure 7. Representation of the sum of the reflection and transmission coefficient $S(\omega)$ for the circuit in Fig. 1 when $L_{I}=6.506 \mathrm{nH}, C_{I}=C_{3}=0.9888 \mathrm{pF}$ $L_{2}=1.391 \mathrm{nH}, L_{3}=6.3 \mathrm{nH}, C_{2}=4.625 \mathrm{pF}$ and $R_{1}=R_{2}=0$, the small asymmetry generated by $L_{1} \neq L_{3}$ generates a non circular shape easy to be seen on the 3D Smith chart.(Circuit simulated between 0-10 GHz).

\section{CONCLUSION}

It that been shown that the sum of the reflection and transmission parameters of a traditional reciprocal symmetrical two port network circuit [10] moves always on a family of coaxal circles (5) when represented on the Smith chart ( if losses are symmetrical at the input and output ports), unit circle when lossless. Alternatively a different line checking criteria parameter was defined in (7). In order to check the symmetry of a circuit it was thus shown that there is no need in testing the phase of input and output return loss and compare them, nor plotting both input and output return loss on the Smith chart or doing even odd mode tests. For a better visualization the 3D Smith chart was proposed. The results can help the microwave engineer check fast the electromagnetic symmetry of the frequency responses of the structures when circuits are lossles or when the losses occur at the input and output ports symmetrically.

\section{ACKNOWLEDGMENT}

This work has been founded by SIWTUNE Marie Curie CIG no 322162, POSDRU/159/1.5/S/134398 and DGCYT MTM2012-33073 grants. A.A.M thanks to Dr. M. Eberspächer for the help.

\section{REFERENCES}

[1] A.A. Muller, P. Soto, D. Dascalu, D. Neculoiu, V.E. Boria, "A 3D Smith chart based on the Riemann Sphere for Active and Passive Microwave Circuits", IEEE Microw. Wirel. Lett. vol 21, no 6, pp 286-288, 2011.

[2] A.A. Muller, P. Soto, D.Dascalu, V.E. Boria " The 3D Smith Chart and It's Practical Applications, Microwave Journal, vol 55, no 7, pp 64-74, 2012.

[3] A.A. Muller, E. Sanabria-Codesal, A. Moldoveanu, V.Asavei, P. Soto, V.E. Boria, S. Lucyszin, "Apollonius Unilateral Transducer Power Gain circles on 3D Smith charts", Electronics Letters, vol 50 no 21, pp 15311533, 2014.

[4] A.A. Muller, P. Soto, A. Moldoveanu. V. Asavei, V.E. Boria, "A Visual Comparison between the voltage and power wave reflection coefficients of microwave circuits", IEEE APMC Conference, Kaohsiung, December, pp 1259-1261, December, 2012.

[5] W. Ciccognani, P. Longhi, S. Colangeli E. Limiti, "Constant Mismatch Circles and Application to Low- Noise Microwave Amplifier Design", IEEE Trans. Microw Theory Techn, vol 61, no 12, pp 1154-1168, 2013.

[6] P. Sjoblom, H Sjoland, "Constant Mismatch Loss Boundary Circles and Their application to Optimum State Dristibution in Adaptive Matching Networks", IEEE Trans.Circuits Syst II, vol 61 no 12, pp 922 - 926, 2014.

[7] B. Mukherejee, P. Prgati, J. Mukherjee, " Hemispherical dielectric resonator antenna based on the Apollonian gasket circles- a fractal approach, IEEE Trans. Antennas Propag, vol 62 no 1, pp 40-48, 2014.

[8] D.A. Brannan, M. Esplen, J.J. Gray, Geometry, Cambridge University Press, 2009

[9] D.A. Frickey, "Conversions between $\mathrm{S}, \mathrm{Y}, \mathrm{Z}, \mathrm{h}, \mathrm{ABCD}$, and T Parameters which are Valid for Complex Source and Load Impedances", IEEE Trans. Microw Theory Techn, vol 42, no 2, pp 205-210, 1994.

[10] H. Mirzaei, G. V. Eleftheriades, "Realizing Non-Foster Reactive Elements Using Negative-Group-Delay Networks", IEEE Trans. Microw Theory Techn, vol 61, no 12, pp 4322-4332, 2013. 